\begin{tabular}{|c|l|}
\hline Title & Focusing of high-frequency dispersive phonons \\
\hline Author(s) & Tamura, S. \\
\hline Citation & $\begin{array}{l}\text { Physical Review B, 25(2), 1415-1418 } \\
\text { https://doi.org/10.1103/PhysRevB.25.1415 }\end{array}$ \\
\hline Issue Date & 1982-01-15 \\
\hline Doc URL & http://hdl.handle.net/2115/5969 \\
\hline Rights & Copyright $\odot$ 1982 A merican Physical Society \\
\hline Type & article (author version) \\
\hline File Information & PRB25-2.pdf \\
\hline
\end{tabular}

Instructions for use 


\title{
Focusing of high-frequency dispersive phonons
}

\author{
S. Tamura \\ Department of Engineering Science, Hokkaido University, Sapporo 060, Japan
}

(Received 7 October 1981)

\begin{abstract}
Focusing of near-zone-edge acoustic phonons in $\mathrm{Ge}$ is studied based on the dynamical matrix of lattice derived by Herman. We find that the focusing behaviors of transverse phonons in the dispersive region are remarkably different from those in the long-wavelength limit and well account for a number of substantial features of the ballistic-phonon images at about $1 \mathrm{THz}$ observed recently by Dietsche $e t a l$.
\end{abstract}

Recently, considerable efforts have been made to understand the propagation properties of large-wavevector dispersive phonons in certain dielectric solids. ${ }^{1-4}$ In particular, Ulbrich et al. have generated near-zone-edge acoustic phonons through the process of energy relaxation of photoexcited $e-h$ pairs in semiconductors of high purity. ${ }^{2}$ They have found that transverse acoustic (TA) phonons propagate in ballistic fashion over lengths of the order of several millimeters. This characteristic of the phonons at low temperatures is in agreement with the theoretical prediction made by Orbach and Vredevoe ${ }^{5}$ that the lifetimes of high-frequency transverse phonons

$\left(\hbar \omega \gg k_{B} T\right)$ against the anharmonic three-phonon interactions are in fact very long limited severely by the energy and momentum conservations. Accordingly, in an ideal sample the large-wave-vector TA phonons are expected to travel macroscopic distances ballistically.

Now, the directional natures of the propagations of such high-frequency phonons will be profoundly influenced by focusing effects due to the anisotropy of lattice, which are considered to be different radically from those in the long-wavelength limit being discussed extensively by many authors. ${ }^{6-11}$ Very recent$\mathrm{ly}$, with the $\mathrm{Pb}$-tunneling junction detector and the phonon imaging method Dietsche et al. have observed the ballistic flux patterns of the near-zoneedge phonons in $\mathrm{Ge}$ of $\mathrm{THz}$ frequencies ( $>700$ $\mathrm{GHz}$ ), which are considerably different from those obtained for longer-wavelength phonons. ${ }^{4}$ In the present Communication, we report that the large features of their findings can be explained in terms of the naive ideas of the phonon focusing modified by the dispersion.

The dispersion curves for $\mathrm{Ge}$ of diamond structure may be characterized by extremely flat TA branches, particularly in the [111] direction, which are also observed in such as GaAs and InSb of zinc-blende type. ${ }^{12}$ In old lattice-dynamical theories of diamondtype crystals, this flattering requires long-range interatomic force constants extending up to fifth- or sixth-nearest neighbors. ${ }^{13}$ At present, the most ap- pealing model for the phonon dispersion curves of group IV elemental crystals with the diamond structure may be the Weber's adiabatic bond charge model $^{14}$ which contains only four or five adjustable parameters. However, in order to save computation time, we shall employ the much simpler dynamical matrix for Ge proposed by Herman ${ }^{13}$ many years ago, which includes the interactions of atoms up to the fifth neighbors. This phenomenological model yields correct frequencies at the midpoints and the edges $X$ and $L$ of the Brillouin zone along the [001] and [111] directions, respectively, but slightly higher frequencies for acoustic modes at the zone edge $K$ in the [110] direction as can be shown right below.

With this model, first we calculated the phonon dispersion curves of two TA branches. The frequencies of the lowest $T 1$ branch at the zone edges $X, L$, and $K$ are 2.53(2.41), 1.98(1.90), and 2.37(2.50) $\mathrm{THz}$, respectively. These values are in good agreement with the recent neutron-scattering data ${ }^{15}$ given in the parentheses. On the other hand, the frequencies of the higher $T 2$ branch at the point $K$ is calculated to be $4.15 \mathrm{THz}$ which is about $17 \%$ larger than the experimental data $3.54 \mathrm{THz}$ at $80 \mathrm{~K} .{ }^{15} \mathrm{Next}$, the constant-frequency surfaces ( $\omega$ surfaces) obtained for the TA phonons of $\mathrm{THz}$ frequencies were drawn. As compared with those of the low-frequency limit, the $\omega$ surfaces are strikingly deformed near cutoff frequencies by the effects of the dispersion, suggesting the drastic changes of the phonon-flux patterns at such high frequencies.

The qualitative understanding for the variation of the flux enhancement of the TA phonons versus frequencies is gained from Figs. 1(a) -1 (c) being displayed for three different frequencies $0.3,0.9$, and $1.5 \mathrm{THz}$, respectively. These diagrams were made by taking a uniform net of wave-vector $(\vec{k})$ directions on the first octant of each $\omega$ surface of the TA modes separated $1^{\circ}$ in both the polar $\left(\theta_{\overrightarrow{\mathrm{k}}}\right)$ and azimuthal $(\phi \overrightarrow{\mathrm{k}})$ angles, and then plotting the associated directions of group velocities $(\vec{v})$ of both the $T 1$ and $T 2$ phonons in a polar projection $\left(\theta_{\vec{v}}, \phi_{\vec{v}}\right)$. It should be noted that the directions falling outside the sector de- 

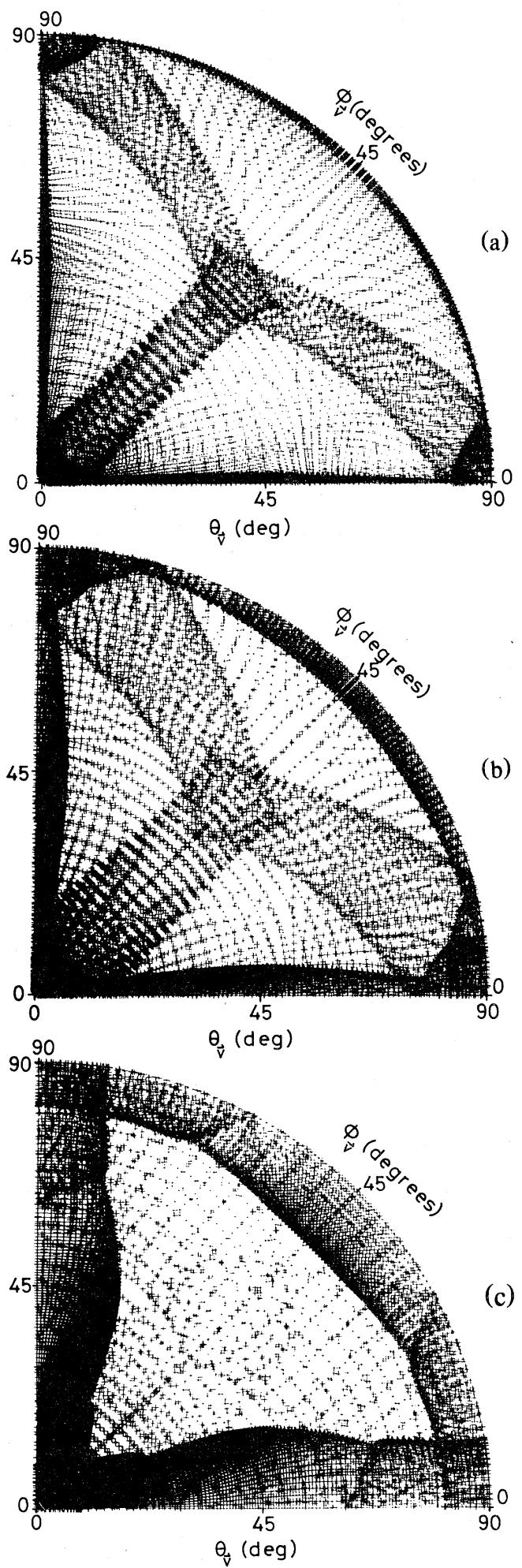

FIG. 1. Focusing of transverse phonons of Ge calculated using the dynamical matrix by Herman (Ref. 13). (a) 0.3 , (b) 0.9 , and (c) $1.5 \mathrm{THz}$. These maps are obtained by overlapping the directional points of the phonons of both the $T 1$ and $T 2$ modes. fined by $0^{\circ} \leqslant \theta_{\vec{v}}, \phi_{\vec{v}} \leqslant 90^{\circ}$ were mapped back in by appropriate symmetry operations. The lines of heavy accumulation of the directional points found in these figures make the folding edges of the group-velocity surface and correspond to singularities of the phonon-focusing factor. ${ }^{16}$ In Fig. 2 the locations of these lines at $0.9 \mathrm{THz}$ are shown to clarify the directions at which the sudden jump of the focusing occurs.

Now, with these plots typical changes of the focusing behaviors with the frequencies may be summarized as follows: The focusing directions which are displayed in the map of the lowest frequency as relatively narrow regions bounded by the folding lines spread with increasing the frequency. At the same time, the flux enhancement in those regions is smeared out producing rather moderate focusing or quasi-isotropic propagation of high-frequency phonons. Such the tendencies are especially notable in the flux pattern of the $T 1$ mode encircling the [111] axis. A characteristic structure of the TA phonon focusing is the coalescences of the folding curves which give rise to cusps in the vicinity of the [111] direction. The existence of the cusp points in the flux enhancement is predicted in the context of the catastrophe theory ${ }^{17}$ applied to the phonons in the three-dimensional lattice with two control parameters $\theta_{\vec{v}}$ and $\phi_{\vec{v}}$. The cusps which can be seen vividly at $0.3-\mathrm{THz}$ frequency are still observed at $0.9 \mathrm{THz}$ though not so significant, but no longer recognized in the map of $1.5 \mathrm{THz}$. We also find that the geometry of the strong focusing regions close to the $\langle 100\rangle$ axes is changed radically with frequencies. More quantitatively, the abrupt amplifications of the $T 1$ phonons in the $(1 \overline{1} 0)$ plane take place at angles $7.5^{\circ}$,

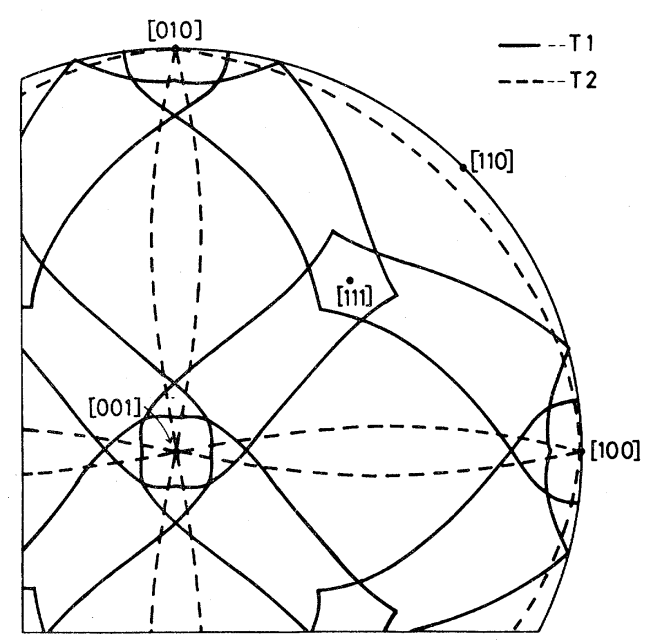

FIG. 2. Lines of heavy accumulation of the directional points. Solid lines represent those of the $T 1$ mode and broken lines the $T 2$ mode. Phonon frequency is $0.9 \mathrm{THz}$. 
$10^{\circ}$, and $13.5^{\circ}$ rotated away from the [001] direction at $0.3,0.9$, and $1.5 \mathrm{THz}$, respectively. It should be noted that the angle at $0.3 \mathrm{THz}$ is almost identical to the value calculated in the continuum model ${ }^{7,11}$ and then we may regard the results obtained at $0.3 \mathrm{THz}$ as prototypes in the nondispersive region.

For the $T 2$ mode the variation of the phonon flux with propagation direction is rather simple. The greater part of the phonons being accumulated in the very vicinity of the (100) and its equivalent planes in the long-wavelength limit are distributed in much wider ranges of real space at $\mathrm{THz}$ frequencies. The angles measured from the [110] axis at which the focusing singularities of the $T 2$ phonons turn up in the (110) plane are found to be $1.5^{\circ}, 5^{\circ}$, and $14^{\circ}$ for $0.3-, 0.9-$, and $1.5-\mathrm{THz}$ frequencies, respectively. More detailed investigation reveals the fact that the complete defocusing area in the map of the $T 2$ mode enclosing the [111] axis (in which no $T 2$ phonon propagation is possible) is magnified in the dispersive region and is separated more distinctly from the strong focusing areas.

All the above mentioned results should be compared with the characteristic features of the phonon images obtained by Dietsche et al. ${ }^{4}$ The image of high-frequency TA phonons $(>0.7 \mathrm{THz})$ detected by $\mathrm{Pb}$-oxide- $\mathrm{Pb}$ tunneling junction is surprisingly sharper than we might expect (revealing the detection of narrow band or quasimonochromatic frequency phonons) and fortunately makes it possible to compare the flux patterns with the calculations. The discernible differences between the tunnel-junction image and the low-frequency pattern obtained by an $\mathrm{Al}$ bolometer are (1) the intensity peaks (the edges of high intensity regions) of the $T 2$ phonons observed in the former are more than twice as far apart from the $\{100\}$ planes as those in the latter, and (2) the shapes of the intensity patterns of the $T 1$ phonons around the $\langle 100\rangle$ axes are much rounder in the high-frequency image. These features are equally observed in Figs. 1 and 2. We would like to emphasize that the disappearance in the image of large-wavevector phonons of the focusing structures extending from the $\langle 100\rangle$ toward $\langle 111\rangle$ axes $^{4}$ is really due to the dispersive effects rather than a shorter mean-free path of the TA phonons by the scattering with isotopes in Ge.

Finally, we shall briefly refer to the focusing of less important longitudinal acoustic (LA) phonons. The whole $\omega$ surface of the LA mode in Ge remains con-

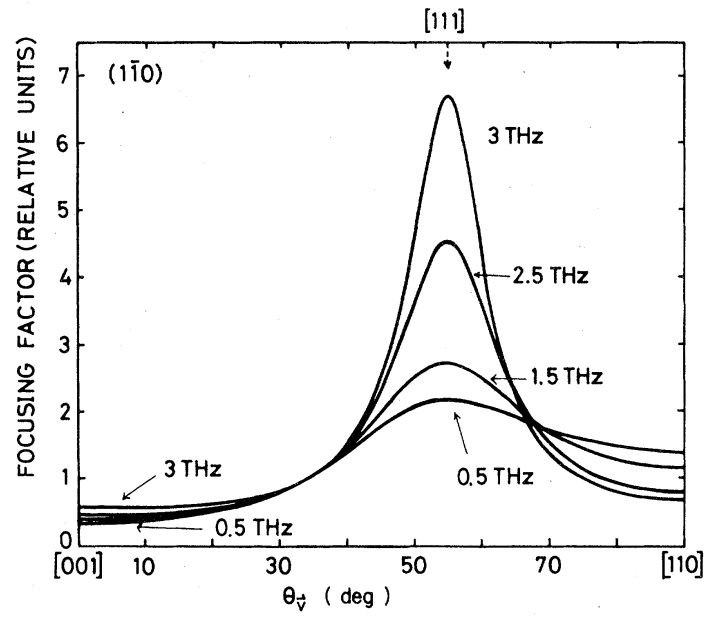

FIG. 3. The flux enhancement of the LA phonons in the (110) plane of Ge.

vex even in the dispersive region if we do not approach so closely the zone boundaries. This means that there exists only one group-velocity vector corresponding to each wave vector on the surface. Hence, with the aid of an analytical expression for the focusing factor generalized to the dispersive region, we can obtain rather easily an enhancement of the LA phonon flux in a specified direction relative to the magnitude it would have in an isotropic medium. Figure 3 shows up to $3-\mathrm{THz}$ frequency the angular dependences of the flux enhancement of the LA phonons

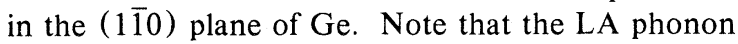
frequency at the point $L$ is $6.60 \mathrm{THz}(6.66 \mathrm{THz})$ by the calculation (experiment ${ }^{15}$ ). In contrast to the results of the TA phonons, the focusing of the LA phonons in the [111] direction is amplified significantly near the zone edge. This strong enhancement of the high-frequency LA phonons in the [111] direction (and also in the [001] direction to be seen at much higher frequencies) should be understood by referring to the shapes of their $\omega$ surfaces near the cutoff frequencies. The section of the $\omega$ surface in the $(1 \overline{10})$ plane is indeed close to a hexagon near the zone edge with the normals of its sides being parallel to the $\langle 111\rangle$ and $\langle 100\rangle$ axes.

The author would like to thank Professor T. Sakuma for a critical reading of the manuscript. This work is supported by a Scientific Research Fund from the Ministry of Education.
${ }^{1}$ W. Grill and O. Weis, Phys. Rev. Lett. 9, 588 (1975).

${ }^{2}$ R. G. Ulbrich, V. Narayanamurti, and M. A. Chin, Phys. Rev. Lett. 45, 1432 (1980).

${ }^{3}$ P. Hu, V. Narayanamurti, and M. A. Chin, Phys. Rev. Lett.
46, 192 (1981).

${ }^{4}$ W. Dietsche, G. A. Northrop, and J. P. Wolfe, Phys. Rev. Lett. 47,660 (1981).

${ }^{5}$ R. Orbach and L. A. Vredevoe, Physics (N.Y.) 1, 92 (1964). 
${ }^{6}$ B. Taylor, H. J. Maris, and C. Elbaum, Phys. Rev. Lett. 23, 416 (1969); Phys. Rev. B 3 , 1462 (1971).

7J. C. Hensel and R. C. Dynes, Phys. Rev. Lett. 43 , 1033 (1979).

${ }^{8}$ G. A. Northrop and J. P. Wolfe, Phys. Rev. Lett. $\underline{43}, 1424$ (1979); Phys. Rev. B 22, 6196 (1980).

${ }^{9}$ P. Taborek and D. L. Goodstein, Solid State Commun. 33, 1191 (1980); Phys. Rev. B 22, 1550 (1980).

${ }^{10}$ A. G. Every, Phys. Rev. B 22,1746 (1980).

${ }^{11}$ S. Tamura and Y. Nakane, Phys. Rev. B $\underline{24}, 4317$ (1981).
${ }^{12} \mathrm{H}$. Bilz and W. Kress, Phonon Dispersion Relations in Insulators (Springer-Verlag, New York, 1979).

${ }^{13}$ F. Herman, J. Phys. Chem. Solids $\underline{8}, 405$ (1959).

${ }^{14}$ W. Weber, Phys. Rev. Lett. 33, 371 (1974); Phys. Rev. B 15,4789 (1977).

${ }^{15}$ G. Nilsson and G. Nelin, Phys. Rev. B 3, 364 (1971).

${ }^{16}$ H. J. Maris, J. Acoust. Soc. Am. 50, 812 (1971).

${ }^{17} \mathrm{See}$, for example, T. Poston and I. Stewart, Catastrophe Theory and its Applications (Pitman, London, 1978). 